\title{
Anestesia en la cirugía laparoscópica abdominal
}

\author{
Jorge Enciso Nano ${ }^{1,2}$ \\ Medico Anestesiólogo, Doctor en Medicina. \\ ${ }^{2}$ Profesor Principal de Anestesiología de la Universidad Nacional Mayor de San Marcos, Perú.
}

\begin{abstract}
Resumen
El desarrollo tecnológico alcanzado permite realizar cirugía de acceso mínimo que con anterioridad requerían grandes incisiones, con elevada morbilidad y traumatismo para el paciente. Junto con la evolución de los medios técnicos e instrumental para este tipo de cirugia, también la anestesiología ha tenido que enfrentar nuevos retos. La monitorización, drogas y el manejo anestésico del paciente han sufrido modificaciones, pues las nuevas variaciones fisiológicas y de complicaciones quirúrgicas han traido consigo cambios importantes en los parámetros hemodinámicos y respiratorios, debidos a la insuflación de la cavidad peritoneal con $\mathrm{CO}_{2}$, el aumento de la presión intraabdominal y los cambios de posición durante el procedimiento.
\end{abstract}

Palabras clave: Morbilidad; parámetros hemodinámicos; insuflación de cavidad peritoneal.

\section{Abstract}

Current technological development allows minimum access surgeries previously requiring big incisions resulting in high patient's morbidity and trauma. Along with the development of technology and instruments for this type of surgery, anesthesiology also faced new challenges. Patient's monitoring, drug and anesthetic management changed because new physiological variations and surgery complications have produced changes in hemodynamic and respiratory parameters, due to $\mathrm{CO}_{2}$ peritoneal cavity insuflation, increased intra-abdominal pressure and changes of position during the procedure.

Key words: Morbidity; hemodynamic parameters; peritoneal cavity insuflation.

An Fac med. 2013;74(1):63-70

\section{INTRODUCCIÓN}

El creciente y rápido avance de la tecnología quirúrgica en las últimas décadas ha permitido la introducción de la cirugía laparoscópica, una revolución quirúrgica muy significativa en la medicina moderna. El espectro de la cirugía laparoscópica es muy amplio, abarcando a la cirugía abdominal, ginecológica, urológica, torácica, ortopédica, entre otras. Ello ha creado nuevos desafíos para la anestesiología moderna que condiciona a una permanente actualización.

En la cirugía laparoscópica se realiza la insuflación de la cavidad abdominal con el gas $\mathrm{CO}_{2}$ (neumoperitoneo), lo cual conlleva a una serie de cambios fisiológicos y complicaciones que no se presentan en la cirugía abierta. El aumento de la presión intraabdominal afecta la fisiología normal de distintos aparatos y sistemas; en su conjunto, a los efectos adversos de la hipertensión intraabdominal en el sistema cardiovascular, respiratorio y renal se les denomina síndrome compartamental abdominal ${ }^{(1,2)}$.

El aumento de la presión intraabdominal trae como consecuencia el incremento de la resistencia vascular periférica, incremento de la presión venosa central, incremento de la presión arterial, aumento de la presión intratorácica, estimulación del sistema nervioso simpático; además, el incremento de la circulación esplácnica se manifiesta con una disminución del flujo arterial hepático, gástrico, renal y mesentérico ${ }^{(1,3)}$. También, el estrés peritoneal libera proteínas de fase aguda en el líquido peritoneal; así, se ha determinado que existe una reacción inflamatoria del fluido peritoneal con el incremento de niveles de interleuquinas IL-1 $\beta$, IL-6, PCR. Estos mediadores son los que inician el proceso inflamatorio, e intervienen en la percepción del dolor y en la posterior formación de adherencias ${ }^{(4,5)}$. Se ha propuesto, a su vez, que la disminución del flujo arterial esplácnico y los cambios hemodinámicos antes descritos son resultado de la afectación del sistema nervioso central, relacionado con un incremento de la presión intracraneal y consecuente liberación de hormonas vasoactivas, con la finalidad de proteger de la isquemia al sistema nervioso ${ }^{(3)}$.

Es así que el neumoperitoneo crea una compleja dinámica de cambio de las condiciones fisiológicas normales, que tienen consecuencias fisiopatológicas durante la laparoscopia. Es fundamental para el anestesiólogo el entendimiento de las consecuencias fisiológicas que se producen en estas condiciones, para evitar o minimizar los efectos deletéreos del $\mathrm{CO}_{2}$ en el paciente. 
En el presente artículo, se hace una revisión de los efectos fisiológicos del neumoperitoneo y sus implicancias en la anestesiología, tanto en la evaluación preanestésica, como en el monitoreo y el postoperatorio.

\section{CAMBIOS FISIOLÓGICOS DURANTE LA ANESTESIA}

Durante la anestesia, en cirugía laparoscópica se desarrollan una serie de cambios fisiopatológicos que dependerán de la insuflación de $\mathrm{CO}_{2}$ dentro de la cavidad abdominal, produciéndose alteraciones hemodinámicas, respiratorias, metabólicas y en otros sistemas los cuales debemos tener en cuenta para su manejo y conocer sus probables complicaciones ${ }^{(6)}$.

El gas que se usa para inducir el neumoperitoneo es el $\mathrm{CO}_{2}$, por su alta solubilidad, gran capacidad de difusión y ser fisiológica y farmacológicamente inerte ${ }^{(7)}$. La cavidad peritoneal está cubierta por una lámina continua intacta de células mesoteliales que tiene una superficie aproximada de $1,5 \mathrm{~m}^{2} \mathrm{y}$ se encuentra cubierto por una delgada película de líquido peritoneal a $37^{\circ} \mathrm{C}$; existe una condición fisiológica normal con un estado de equilibrio homeostático, bioquímico y físico cuando existe una presión intraabdominal de hasta 3 mmHg. Crear y mantener un neumoperitoneo altera esas circunstancias, con los consecuentes efectos físicos, químicos y biológicos ${ }^{(8-11)}$. Entre otros efectos, el aumento de la presión intraabdominal induce respuesta de estrés hemodinámico, altera el retorno venoso de las extremidades inferiores, disminuye el gasto cardiaco y hay un aumento de la presión arterial media así como de la resistencia vascular sistémica ${ }^{(6,12,13)}$. Un neumoperitoneo que mantiene la temperatura corporal, la hidratación del tejido y la integridad durante la cirugía laparoscópica, mejora el resultado clínico.

Una presión abdominal de hasta 12 $\mathrm{mmHg}$ ha demostrado ser la adecuada para la mayoría de procedimientos qui- rúrgicos, minimizando los efectos adversos; con dicha presión, en pacientes jóvenes y sin comorbilidad, la retención de $\mathrm{CO}_{2}$ es mínima. Se ha observado cambios hemodinámicos significativos cuando la presión intraabdominal se eleva por encima de $12 \mathrm{mmHg}$; así, en un estudio observacional prospectivo se observó los siguientes cambios hemodinámicos: disminución del volumen de eyección, disminución del gasto cardiaco, así como un aumento de la resistencia vascular sistémica ${ }^{(14)}$. En una revisión Cochrane se evaluó los daños y beneficios de la baja presión del neumoperitoneo $(<12 \mathrm{mmHg})$ en comparación con la presión estándar (12 $\mathrm{mmHg}$ ) en pacientes sometidos a colecistectomía laparoscópica; no se halló diferencias significativas en cuanto a mortalidad, complicaciones pulmonares, complicaciones postoperatorias o conversión a colecistectomía abierta. Debido a sesgos de estudio de algunos ensayos de la revisión y dado que solo se estudió a pacientes sometidos a colecistectomía, es incierta aún la seguridad de utilizar presiones intraabdominales bajas $(<12 \mathrm{mmHg})^{(15)}$.

En la cirugía laparoscópica, la anestesia general es el método de elección, ya que permite al anestesiólogo un control preciso de la ventilación y modificar los parámetros ventilatorios, en base en las alteraciones que puedan presentarse. Supone algunas ventajas, como el adecuado control de la respiración, óptima protección de la vía aérea, excelente relajación muscular, monitoreo del dióxido de carbono al final de la espiración con el capnógrafo, entre otras ${ }^{(16-18)}$. Algunas complicaciones con el método de anestesia general, así como algunas características individuales del paciente (comorbilidad), posibilitan el empleo de la anestesia regional para ciertos casos ${ }^{(19,20)}$. En los pacientes con riesgo anestésico III - IV ASA (Sociedad Americana de Anestesiología), no solo serán necesarias la oximetría de pulso, capnografía, cardioscopia y la tensión arterial, sino además se requiere de monitorización invasiva (ecocardiografía transesofágica, presión venosa central, entre otras) para tener un control adecuado de las variaciones fisiológicas o complicaciones ${ }^{(21)}$.

\section{Alteraciones hemodinámicas}

Los cambios hemodinámicos observados durante la cirugía laparoscópica van a estar determinados por los cambios de posición a que están sometidos los pacientes y por el efecto mecánico que ejerce la comprensión del $\mathrm{CO}_{2}$ dentro de la cavidad peritoneal. Durante la inducción anestésica, las presiones de llenado del ventrículo izquierdo disminuyen, provocando a su vez una disminución del índice cardiaco, manteniendo igual la presión arterial media. Estos cambios son debidos probablemente a la acción depresora de los fármacos inductores, como también por la disminución del retorno venoso por la posición del paciente ${ }^{(22)}$. Al comenzar la insuflación del peritoneo con $\mathrm{CO}_{2}$, se va a producir un aumento de la presión arterial, tanto sistémica como pulmonar, lo cual provoca una disminución del índice cardiaco, manteniendo igual la presión arterial media. La distensión del peritoneo provoca la liberación de catecolaminas que desencadenan una respuesta vasoconstrictora. Hay elevación de presiones de llenado sanguíneo durante el neumoperitoneo, debido a que el aumento de la presión intrabdominal provocará una redistribución del contenido sanguíneo de las vísceras abdominales hacia el sistema venoso, favoreciendo un aumento de las presiones de llenado. También se ha observado una disminución del flujo venoso femoral, cuando aumenta la presión intrabdominal por hiperinsuflación. Como consecuencia hay disminución del retorno venoso y la caída de la precarga cardiaca. En resumen, durante el inicio del neumoperitoneo existe un aumento de las resistencias vasculares sistémicas y pulmonares (figura 1). Otros estudios han demostrado que durante la insuflación del neumoperitoneo habrá un aumento del trabajo cardíaco y consumo miocárdico de oxígeno ${ }^{(23-25)}$. 


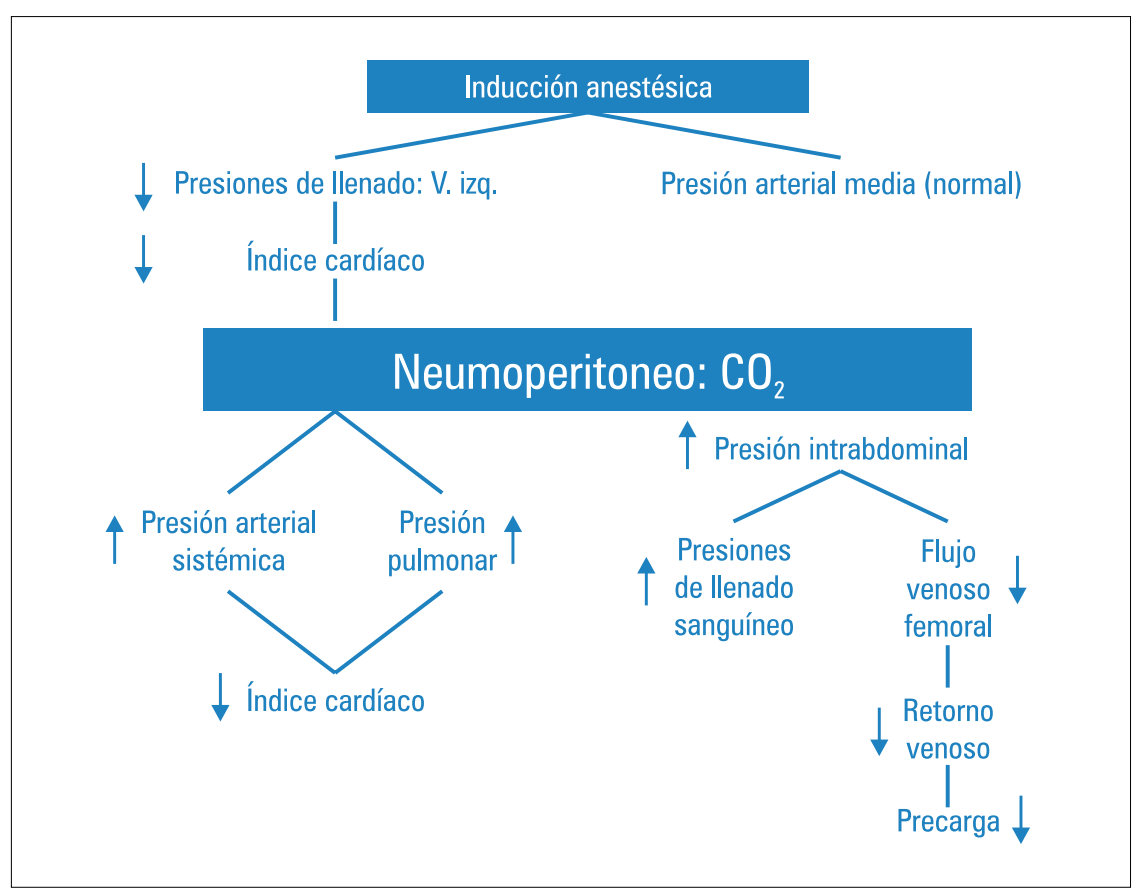

Figura 1. Alteraciones hemodinámicos durante la anestesia.

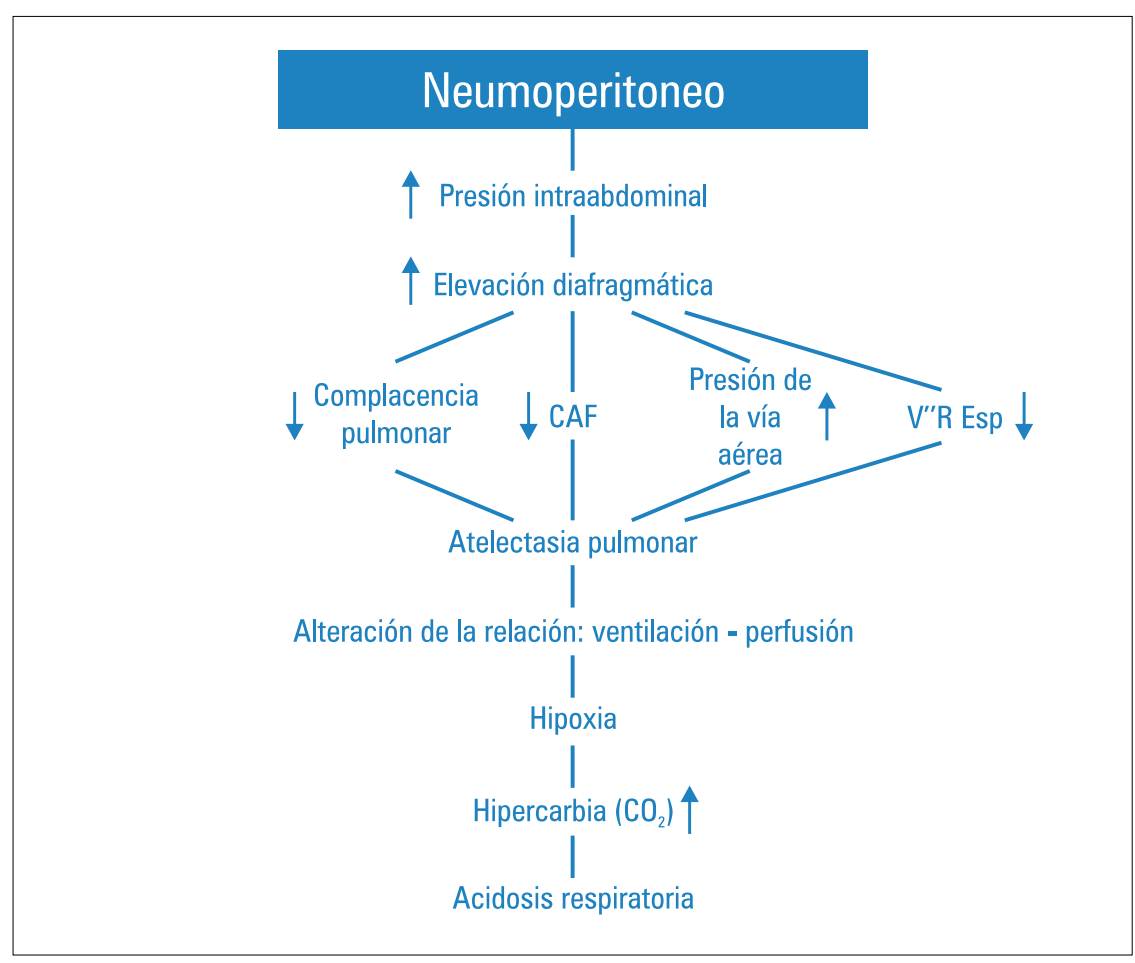

Figura 2. Alteraciones respiratorias durante la anestesia

\section{Alteraciones respiratorias}

La insuflación de $\mathrm{CO}_{2}$ en la cavidad abdominal y el aumento de la presión intrabdominal provocada por el neumoperitoneo son factores que influyen de manera particular en la función pulmonar. Se ha demostrado que durante la laparoscopia se produce una disminución de la complacencia (compliance) pulmonar, del volumen de reserva respiratorio y de la capacidad residual funcional, con el aumento de la presión de pico inspiratoria ${ }^{(26)}$. Como consecuencia, se produce una redistribución de flujo a zonas pobremente prefundidas durante la ventilación mecánica, con el aumento del shunt intrapulmonar y del espacio muerto. También, se ha observado un aumento en la gradiente de presión arterial de $\mathrm{CO}_{2}\left(\mathrm{PaCO}_{2}\right)$ presión espirada de $\mathrm{CO}_{2}\left(\mathrm{PETCO}_{2}\right)$, con disminución del $\mathrm{pH}$. Esta alteración puede ser corregida aumentando el volumen minuto entre 15 y $20 \%$ y utilizando PEEP de $5 \mathrm{~cm}$ de $\mathrm{H}_{2} \mathrm{O}$. Existe también un aumento de la presión pico y la presión meseta, que luego se estabilizarán. Cuando se utiliza la posición de Trendelemburg en pacientes con cirugía ginecológica en ventilación espontánea, la presión abdominal así como el desplazamiento de las vísceras en sentido cefálico ejercen presión sobre el diafragma, dificultando la respiración, dando como resultado taquipnea e hipercarbia ${ }^{(27)}$.

Referente a la absorción del $\mathrm{CO}_{2}$ por el peritoneo, al parecer esta se estabiliza después de los primeros $10 \mathrm{minu}$ tos de haber aumentado la presión intrabdominal ${ }^{(28)}$. Se dice que la presión que ejerce el neumoperitoneo sobre los capilares peritoneales actúa como un mecanismo protector, impidiendo la absorción de $\mathrm{CO}_{2}$ a través de este. Al final del procedimiento, cuando disminuye la presión intrabdominal por la salida del $\mathrm{CO}_{2}$, vamos a encontrar una mayor frecuencia de absorción de $\mathrm{CO}_{2}$ que puede ser registrada mediante la capnografía (figura 2). 


\section{Alteraciones de la función renal}

El aumento de la presión intrabdominal produce una elevación de la presión venosa renal, la cual genera incremento de la presión capilar intraglomerular. En consecuencia, disminuye la presión de perfusión renal. Se ha detectado una disminución del flujo plasmático renal (FPR) y de la tasa de filtración glomerular (figura 3).

En los casos de insuficiencia renal y ante laparoscopias prolongadas, puede haber deterioro de la función renal. El aumento de la presión intrabdominal no afecta la función de los túbulos de intercambio iónico, aclaración y absorción de agua libre ${ }^{(29,30)}$.

\section{Reflujo gástrico}

El incremento de la presión intraabdominal que se produce con el neumoperitoneo puede ser suficiente para elevar el riesgo de reflujo pasivo del contenido gástrico ${ }^{(31)}$ (figura 4).

Los pacientes que tienen antecedentes de diabetes complicada con gastroparesia, hernia hiatal, obesidad o algún tipo de obstrucción de la salida gástrica son los más propensos al aspirado de contenido gástrico.

\section{Efectos del carboperitoneo en la} fisiología del sistema nervioso central

EL neuroperitoneo va a producir elevación de la presión intrabdominal y elevación diafragmática; esto conlleva a la hipoxia, hipercarbia. La hipercarbia va a producir vasodilatación cerebral, aumentando el flujo sanguíneo cerebral y por ende aumento de la presión intracraneal ${ }^{(32)}$ (figura 5).

\section{Efectos del carboperitoneo en el hígado}

El neumoperitoneo provocado va a producir una reducción del flujo venoso portal, trayendo como consecuencia hipoperfusión hepática, que puede producir lesión aguda del hepatocito si el tiempo que dura el acto operatorio es muy prolongado ${ }^{(33)}$.

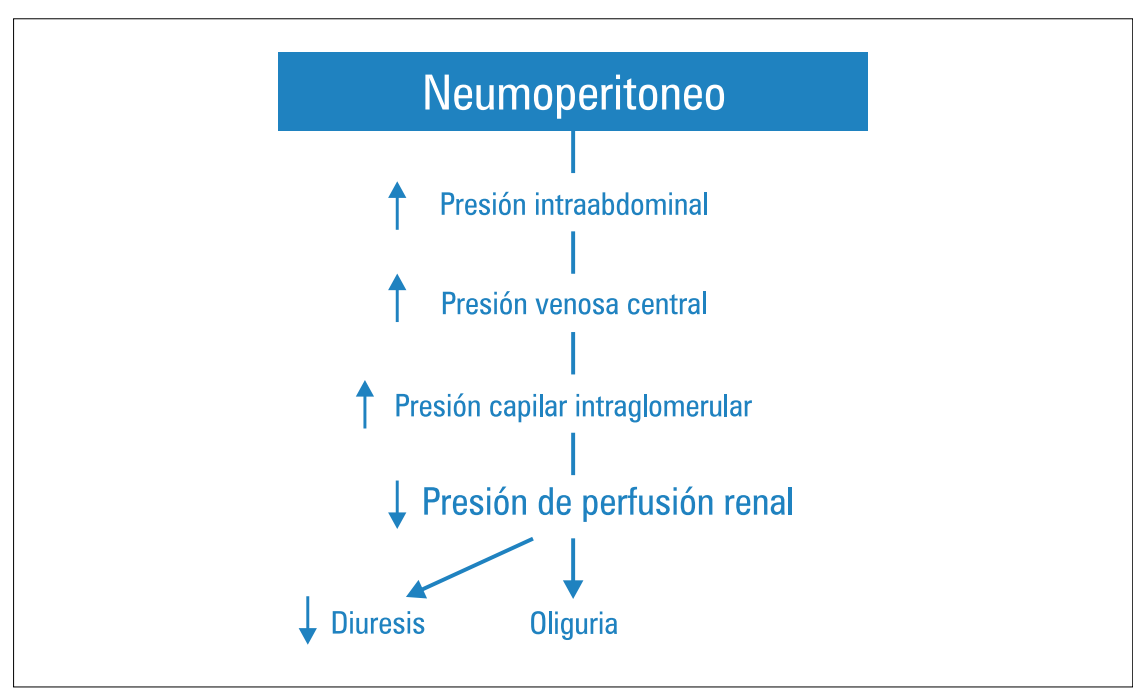

Figura 3. Alteraciones de la función renal durante la anestesia.

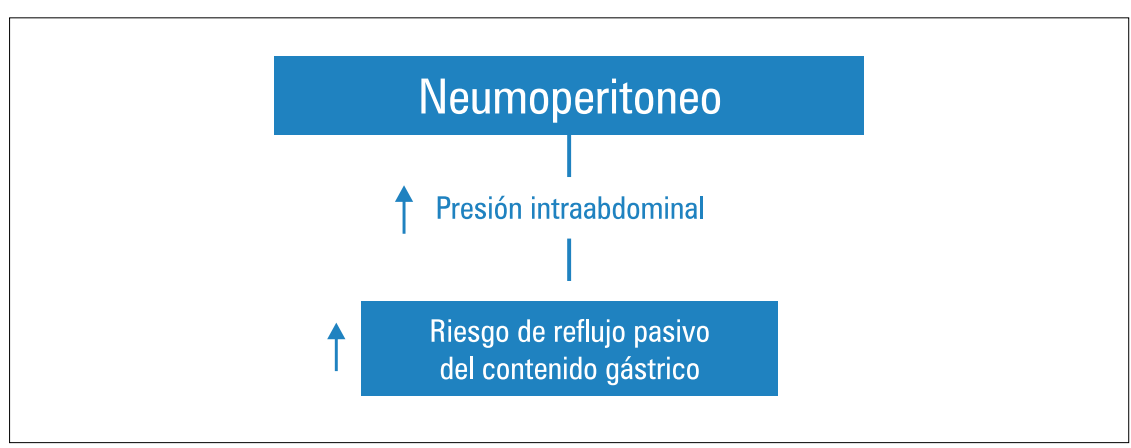

Figura 4. Cambios fisiológicos durante la anestesia: reflujo gástrico.

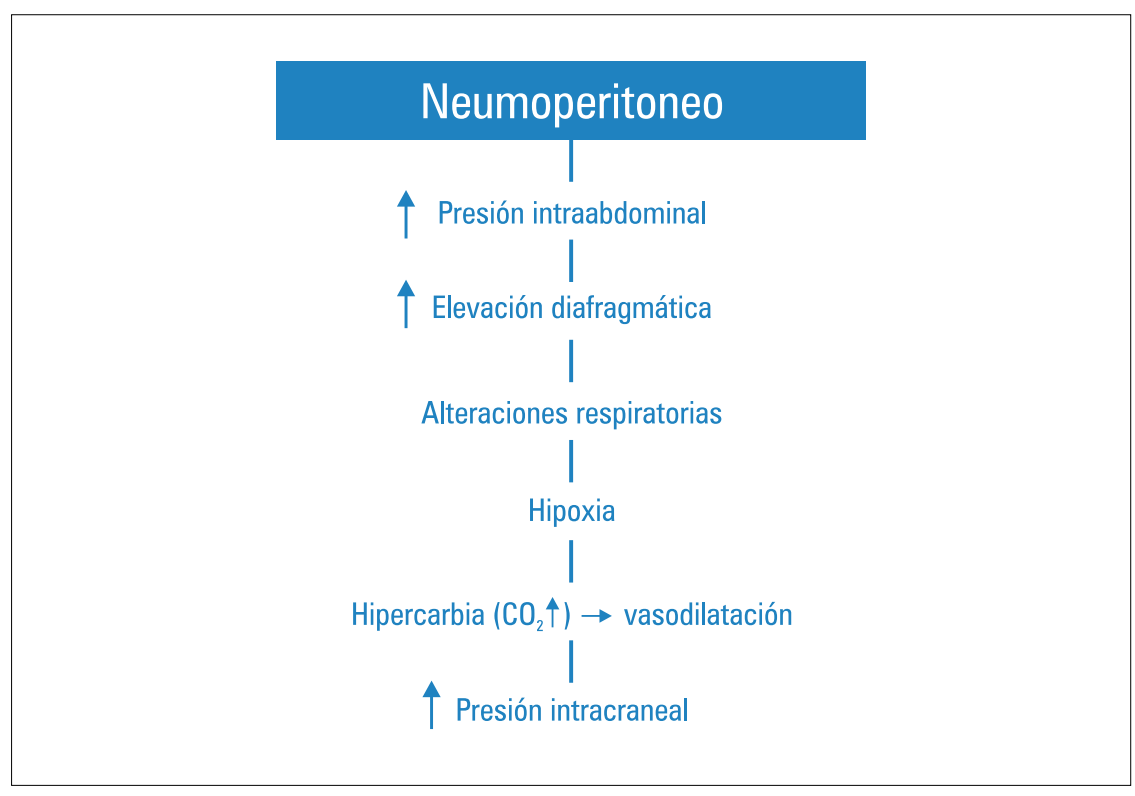

Figura 5. Efectos del carbioperitoneo en la fisiologia del sistema nervioso central. 


\section{MANEJO ANESTÉSICO DE LA CIRUGÍA LAPAROSCÓPICA}

\section{Evaluación pre anestésica}

- Permite evaluar el estado físico clínico anestesiológico del paciente (ASA). Se debe advertir de la incidencia elevada de la morbilidad postoperatoria (dolor, náusea, vómito).

- La evaluación preanestésica permitirá poner en evidencia las patologías cardiorrespiratorias, que constituyen una contraindicación absoluta o relativa, según el grado de insuficiencia.

- Los estados de shock séptico o hipovolémico son considerados como contraindicaciones absolutas.

- La obesidad no es contraindicación absoluta, pero los anestesiólogos recomiendan hacerles a estos pacientes una evaluación cardiovascular y respiratoria previa al acto anestésico.

- Los antecedentes quirúrgicos abdominales que limiten la realización del acto deben ser apreciados por el cirujano.

- Las contraindicaciones de la posición de Trendelemburg, principalmente los estados de hipertensión endocraneana e intervenciones o traumatismos craneanos recientes y el glaucoma agudo deben ser respetados.

- La presencia de enfermedad pulmonar preexistente sugiere la necesidad de una evaluación especializada que incluya AGA y prueba de función pulmonar previa a la operación, que permitan evaluar los riesgos de hipercarbia y acidosis respiratoria $y / o$ hipoxemia severa descritos en estos pacientes.

- Se debe también investigar patologías gastrointestinales susceptibles de aumentar el residuo gástrico o el riesgo de reflujo, lo cual haría necesaria la administración de medicamentos que aumenten el tono del esfínter esofágico.
- Una consideración especial debe tenerse en el segundo y tercer trimestre del embarazo, donde las repercusiones hemodinámicas de la hiperinflación abdominal serían de mayor consecuencia en estas etapas de gestación, por lo cual no se recomienda este tipo de procedimiento.

\section{Medicación preanestésica}

Teniendo en cuenta que la cirugía laparoscópica es un procedimiento que se viene empleando por su abordaje mínimamente invasivo, favoreciendo una pronta recuperación y el reintegro a las actividades diarias del paciente, debemos entonces utilizar la medicación preanestésica adecuada que no prolongue la recuperación del paciente. Por lo tanto, drogas que puedan prolongar la estadía del paciente en recuperación están relativamente contraindicadas, como por ejemplo los opiáceos de larga duración tipo fentanyl, ya que además de prolongar el efecto anestésico puede producir prurito y náuseas en el postoperatorio. Sin embargo, queda a criterio del anestesiólogo su utilización. Para la ansiólisis, es más recomendable utilizar benzodiacepinas, en especial el midazolam ${ }^{(34)}$, por su rápido metabolismo y efectos amnésicos. Referente a las náuseas y vómitos postoperatorios, se ha revisado una cantidad suficiente de literatura en donde se propone el uso de diferentes drogas para su prevención. El droperidol sigue siendo un potente antiemético, teniendo en cuenta que dosis muy altas producen efectos indeseables como el extrapiramidalismo. El ondancetron, que es un antagonista específico de los receptores serotoninérgicos tipo III, ha demostrado su eficacia antiemética ${ }^{(35)}$ en cirugía laparoscópica. Recientemente, se ha demostrado que el empleo de un glucocorticoide tipo dexametasona tiene acción antiemética y está siendo empleando en algunos hospitales y clínicas ${ }^{(36)}$.

\section{Monitoreo}

El monitoreo durante la cirugía laparoscópica debe ser lo suficientemen- te efectivo para detectar los cambios tanto hemodinámicos como respiratorios antes descritos, así como también alertar sobre posibles complicaciones. El monitoreo debe incluir cardioscopio para la frecuencia y ritmo cardiaco, presión arterial con esfigmomanómetro electrónico, con ciclos de tiempo ajustables. En lo referente al monitoreo respiratorio, es indispensable la observación de las presiones dentro de las vías respiratorias, que por lo general pueden verse en los manómetros de presión de las máquinas de anestesia. De todos los monitores para la cirugía laparoscópica, quizás el más importante sea el capnógrafo, pues medirá la presión espiratoria de $\mathrm{CO}_{2}$ del paciente, el estado metabólico; nos sirve como monitoreo de desconexión y, lo más importante, nos alertará sobre la absorción inadvertida de $\mathrm{CO}_{2}$. No se justifica el no monitorizar el $\mathrm{CO}_{2}$ durante la cirugía laparoscópica, debido a que al estar insuflando $\mathrm{CO}_{2}$ al paciente a través de una máquina, el riesgo de embolismo por $\mathrm{CO}_{2}$ y absorción del mismo estará siempre latente y la única forma de detectarlo es mediante el capnógrafo. Otros monitores que podemos emplear serán la oximetría de pulso, monitor de relajación muscular y de gases expirados.

- Estimulador de nervio periférico: nos permite evaluar el grado de relajación muscular, para mantenerla en niveles adecuados durante el procedimiento, y así poder ventilar mejor al paciente y permitir un mejor campo quirúrgico.

- Manómetro de presión de vía aérea: es importante monitorizar la presión de la vía aérea, para detectar aumentos exagerados, lo que apuntaría a una probable complicación pulmonar. En general, el cambio de presión pre y postinsuflación no debe ir más allá de 5 a 10 centímetros de agua.

- Sondas orogástrica o nasogástrica: una vez garantizada la permeabilidad de las vías respiratorias mediante la intubación orotraqueal, se coloca una sonda orogástrica para descomprimir el estómago al extraer 
su contenido, con lo que se reducirá el riesgo de punción visceral durante el neumoperitoneo, mejorará la visualización laparoscópica y se volverá mínimo el riesgo de aspiración.

- También será de importancia la presión arterial no invasiva y el control de la temperatura.

\section{Técnica anestésica}

La técnica anestésica a emplear dependerá de la experiencia del anestesiólogo, el tipo de intervención y siempre tomando en cuenta los cambios fisiopatológicos que se producen durante la introducción de $\mathrm{CO}_{2}$ para realización del neumoperitoneo ${ }^{(37)}$. Para la colecistectomía laparoscópica, por ejemplo, la mayoría de los anestesiólogos prefiere la anestesia general inhalatoria con ventilación controlada, de manera tal que si aumenta la presión de $\mathrm{CO}_{2}$ respiratoria, se pueda hiperventilar al paciente para 'barrer' el $\mathrm{CO}_{2}$. Sin embargo, algunos autores tienen experiencia con la máscara laríngea y la ventilación espontánea; en estos casos se sopesará el riesgo de broncoaspiración y retención de $\mathrm{CO}_{2}$. Otros autores preconizan el uso de bloqueos regionales a niveles altos para este tipo de cirugías; sin embargo, las complicaciones de este procedimiento utilizando sedación son mayores debido al riesgo de hipoventilación, además del dolor reflejo que se produce por distensión frénica. Otras indicaciones de anestesia regional serían en el área ginecológica, como esterilización, bridas, endometriosis y otros. En estos procedimientos, el neumoperitoneo no alcanza presiones tan altas que afecten la ventilación y la hemodinamia del paciente. Actualmente, se considera que, en cirugía laparoscópica, es mandatorio y correcto administrar anestesia general inhalatoria con el paciente intubado y suministrándole ventilación controlada, utilizando un ventilador mecánico.

La anestesia general con intubación y ventilación controlada previenen el riesgo de hipoventilacion y. regurgitación ligada a la presión intradominal incrementada y a la postura.
La ventilación controlada limita las perturbaciones cardiorrespiratorias ligadas a la laparoscopia. Un $\mathrm{FiO} 2$ de 0,3 a 0,5 permite la prevención de hipoxia.

Una hiperventilación moderada (volúmenes tidales mayores y/o aumento de la frecuencia respiratoria en $50 \%$ superior a las necesidades básicas) previene la hipercapnea. Los anestésicos con acción básico dilatadora pueden ser utilizados para minimizar los efectos hemodinamicos, así como también evitar los agentes depresores miocárdicos.

Referente a las drogas inductoras, no existe alguna preferencia, ya que se puede utilizar el tiopental sódico como el propofol, siempre y cuando no exista contraindicación para alguno de ellos ${ }^{(38)}$. Se puede utilizar relajantes musculares de acción intermedia y corta, como el bromuro de vecuronio, besilato de atracurio o bromuro de rocuronio. Sin embargo, hay que tener cuidado con el atracurio, por desencadenar liberación de histamina. Si se desea usar opiáceos, se debe usar los de acción corta, como el alfentanyl. Para el mantenimiento de la anestesia, se puede utilizar los halogenados recomendables para la cirugía ambulatoria, como el isoflurano, sevoflurano o desflurano. Hay que tener en cuenta que una vez se intube al paciente colocar una sonda nasogástrica para descomprimir el estómago, pues muchas veces la distensión gástrica dificulta visualizar las vísceras abdominales.

En relación al óxido nitroso (N2O), su uso en laparoscopia sigue siendo muy controversial, aunque no está contraindicado ${ }^{(39)}$. Se dice que como es más difusible tenderá a aumentar la presión abdominal; otros lo relacionan con la persistencia del dolor en el postoperatorio. En la medida de lo posible, se tenderá a realizar el mantenimiento de la anestesia con oxígeno y aire para evitar complicaciones.

\section{Fin de la laparoscopia}

La exsuflación del neumoperitoneo debe ser lenta; un aflujo brutal de sangre rica en metabolitos hacia la circu- lación central, realiza una verdadera embolia ácida. El retorno a la posición horizontal debe ser progresivo para asegurar reperfusión homogénea y prevenir hipotensión.

\section{Repercusión}

Se debe buscar el retorno progresivo a una situación hemodinámica y ventilación anteriores. La prevención y tratamiento de morbilidad postoperatoria (dolor, náuseas, vómitos), deben ser tenidos en cuenta. Se recomienda metoclorpropamida (Primperán) 10 a $20 \mathrm{mg}$ $\mathrm{EV}$, diclofenaco $75 \mathrm{mg} \mathrm{IM}$, poco antes del fin de la laparoscopia.

\section{COMPLICACIONES DE LA CIRUgía LAPAROSCÓPICA}

Las complicaciones relacionadas a la anestesia ocurren entre 0,016 a un $0,075 \%$ en los pacientes y con muy poca frecuencia son fatales. Las complicaciones pueden ser divididas en aquellas debidas a la técnica anestésica, a la insuflación de $\mathrm{CO}_{2} \mathrm{o}$ a error en las técnicas e instrumental quirúrgico ${ }^{(40-44)}$.

\section{Náuseas y vómitos}

La manipulación del peritoneo parietal y de las vísceras abdominales luego del neumoperitoneo puede producir una estimulación vagal que desencadenará los reflejos de náuseas, diaforesis y bradicardia. Es por este motivo que debemos tener en cuenta la medicación con ondancetron, droperidol y bloqueantes $\mathrm{H} 2$ previo a la cirugía.

\section{Dolor}

Aunque una de las ventajas de la cirugía laparoscópica es la disminución del dolor, esta complicación suele presentarse luego de este tipo de procedimiento. Posterior a la cirugía el $\mathrm{CO}_{2}$ tiende a acumularse en los espacios subdiafragmáticos irritando el nervio frénico; este, por metámeras, provocará un dolor a nivel de los hombros y la espalda, del cual se quejan los pacientes. Este dolor suele calmar espontáneamente luego 
de varias horas, mientras se absorbe el $\mathrm{CO}_{2}$. Sin embargo, para aliviar la queja del paciente se ha empleado analgésicos no esteroideos, como el ketoprofeno, el ketorolaco, entre otros. Una técnica preconizada por algunos anestesiólogos es la de dar oxígeno $100 \%$ media hora después de haberse retirado el neumoperitoneo, para así estar seguros que no quede gas carbónico en la cavidad peritoneal.

Algunos autores recomiendan hacer instilaciones subdiafragmáticas de bupivacaína $0,25 \%$. Un buen esquema terapéutico para el dolor puede ser aplicar $75 \mathrm{mg}$ de diclofenaco IM o EV después de la inducción anestésica, posteriormente diclofenaco cada 12 horas IM; también, 50 mg de tramadol.

\section{Traumatismos}

Los traumatismos a los cuales nos referimos depende de la técnica del cirujano: traumatismos viscerales, hemorragias, punciones de vasos o vísceras sólidas, que ocasionan enfisema subcutáneo, neumoretroperitoneo, neumotórax, neumomediastino y neumopericardio.

\section{Embolismo gaseoso}

$\mathrm{El} \mathrm{CO}_{2}$ generalmente es absorbido a través de la superficie peritoneal y disuelto en la sangre venosa. Ocasionalmente el gas puede ser introducido en una arteria o vena mediante una punción accidental de un vaso sanguíneo ${ }^{(45)}$. De esta manera se produce un embolismo gaseoso, cuya incidencia es de 0,002 a 0,016\%. El gas embolizado rápidamente llega a la vena cava y a la aurícula derecha obstruyendo el retorno venoso, disminuyendo el gasto cardiaco y la presión arterial sistémica. El embolismo de $\mathrm{CO}_{2}$ produce cambios bifásicos en la capnografía; inicialmente hay un aumento de $\mathrm{CO}_{2}$, porque se está excretando el disuelto en la sangre. Posteriormente, se observa una disminución del mismo, debido a un aumento del espacio muerto como consecuencia de la obstrucción de las arteriolas por las burbujas de aire. Los signos clínicos del embolismo aéreo durante la laparosco- pia incluyen una repentina y profunda hipotensión, cianosis, taquicardia, arritmias y alteraciones de los ruidos cardiacos, los cuales se hacen tipo fonéticos, además de conseguirse el signo de 'rueda de molino' a la auscultación mediante estetoscopio esofágico.

\section{Enfisema subcutáneo}

Esto se observará en el abdomen, tórax, cuello y cara y hacia abajo a la región inguinal y aun genitales. Se debe a filtración de $\mathrm{CO}_{2}$ a través de las punciones, lo que diseca y difunde hacia territorios más laxos ${ }^{(46)}$. Al existir enfisema subcutáneo habitualmente se produce un aumento de los niveles sanguíneos de $\mathrm{CO}_{2}$ (hipercarbia) que es conveniente corregir. Por lo general, el enfisema desaparece en corto tiempo, por la alta difusibilidad del $\mathrm{CO}_{2}$.

\section{Neumotórax}

Se debe sospechar los pacientes portadores de enfisema pulmonar o con bulas enfisematosas y en aquellos con defectos congénitos diafragmáticos, como el hiatus pleuroperitoneal, hiatus esofágico y otros, que permitieron el paso del dióxido de carbono a la pleura. En el cuadro clínico se puede encontrar caída de la presión arterial, cianosis, taquicardia o bradicardia, enfisema subcutáneo, dificultad en la ventilación, hiperresonancia a la percusión del tórax y ruidos respiratorios aumentados o disminuidos a la auscultación. Para prevenir el neumotórax se recomienda que la presión intrabdominal no sobrepase los $20 \mathrm{mmHg}$ y utilizar como gas a difundir el $\mathrm{CO}_{2}$ ya que es altamente difusible.

\section{Colapso cardiovascular}

Se evidencia por hipoxemia, cianosis, bradicardia, hipotensión severa seguida de paro cardíaco. En general, se podría explicar por hemorragias estimulación del vago por tracción del peritoneo al realizar el neumoperitoneo, o por embolias de $\mathrm{CO}_{2}$, que ingresaron por canales venosos abiertos.
A pesar de lo anteriormente descrito, la mortalidad debido a la laparoscopia es baja y continua disminuyendo hasta cifras de 1/100 000 .

Entonces, cuando el anestesiólogo informa que el paciente presenta un colapso cardiovascular o respiratorio es recomendable evacuar inmediatamente el neumoperitoneo, hasta que se pueda estabilizar el estado hemodinámico del paciente y luego continuar con la cirugía laparoscópica o proceder a la conversión del procedimiento en laparotomía abierta.

\section{Arritmias}

Existen reportes que indican que el $17 \%$ de las laparoscopias presenta arritmias cardíacas cuando se usa $\mathrm{CO}_{2}$ para el neumoperitoneo, como bradicardia, taquicardia, extrasístoles supraventriculares y ventriculares. La bradicardia es la arritmia más frecuente en relación al inicio del neumoperitoneo y se explica por la reacción vagal ocasional por la distensión del peritoneo. Las otras arritmias se explican por la producción de hipercarbia.

\section{Cambios de temperatura: hipotermia}

El descenso de la temperatura corporal se debe a la anestesia general y a la insuflación del gas $\left(\mathrm{CO}_{2}\right)$. El $\mathrm{CO}_{2}$ se vuelve más frío cuando es liberado en la cavidad abdominal. La expansión súbita del $\mathrm{CO}_{2}$ baja la temperatura corporal, por esta razón el monitoreo de la temperatura es recomendado en los procedimientos laparoscópicos de larga duración.

\section{AGRADECIMIENTOS}

Al Dr. José Ramos, por su colaboración en el perfeccionamiento del manuscrito.

\section{REFERENCIAS BIBLIOGRÁFICAS}

1. Togal T, Gulhas N, Cicek M, Teksan HI, Ersoy O. Carbon dioxide pneumothorax during laparoscopic surgery. Surg Endosc. 2002;16(8):1242. 
2. Volz J, Koster S, Spacek Z, Paweletz N. Characteristic alterations of the peritoneum after carbon dioxide pneumoperitoneum. Surg Endosc. 1999;13:611-14.

3. Brasesco OE, Szomstcin S, Mailapur RVR, et al. La fisiopatología del pneumoperitoneo. Diez años de estudio en busca de una teoría unificadora. Rev Mex de Cir Endosc. 2002;3:101-6.

4. Jiang J DU, Pei-Wu PW Yu. Effect of different $\mathrm{CO}(2)$ pneumoperitoneum on IL-1 $\beta$ and IL-6 in abdominal cavity. Zhonghua Wei Chang Wai Ke Za Zhi. 2012;15(8):834-6.

5. Ott D. Reduced Peritoneal inflammation using wet gas compared to cold dry gas as measured by C-reactive protein and interleukin-6. JSLS. 2003;7:S1.2.

6. Henny CP, Hofland J. Laparoscopic surgery: pitfalls due to anesthesia, positioning, and pneumoperitoneum. Surg Endosc. 2005;19(9):1163-71.

7. Leighton TA, Liu S, Bongard FS. Comparative cardiopulmonary effects of carbon dioxide versus helium pneumoperitoneum. Surgery. 1993:113:527-31.

8. Albanese A, Albanese E. Mino J, Gomez E, Gomez M, Zandomeni M, Merlo A. Peritoneal surface area: measurements of 40 structures covered by peritoneum: correlation between total peritoneal surface area and the surface calculated by formulas. Surg Radiol Anat. 2009;31:369-77.

9. Ott DE. Desertification of the peritoneum by thin-film evaporation during laparoscopy. JSLS. 2003;7:189-95.

10. Sammour T, Kahokehr A, Hill A. Meta-analysis of the effect of warm humidified insufflation on pain after laparoscopy. Br J Surg. 2008;95:950-6.

11. Binda M, Molinas C, Hansen P, Koninckx P. Effect of desiccation and temperature during laparoscopy on adhesion formation in mice. Fertil Steril. 2006;86:166-75.

12. Galizia G, Prizio G, Lieto E, Castellano P, Pelosio L, Imperatore V, et al. Hemodynamic and pulmonary changes during open, carbon dioxide pneumoperitoneum and abdominal wall-lifting cholecystectomy. A prospective, randomized study. Surg Endosc. 2001;15(5):477-83.

13. Joris JL, Chiche JD, Canivet JL, Jacquet NJ, Legros JJ, Lamy ML. Hemodynamic changes induced by laparoscopy and their endocrine correlates: effects of clonidine. J Am Coll Cardiol. 1998;32(5):1389-96.

14. Borg IR Mertens zur, Lim A, Verbrugge SJ, IJzermans JN, Klein J. Effect of intraabdominal pressure elevation and positioning on hemodynamic responses during carbon dioxide pneumoperitoneum for laparoscopic donor nephrectomy: a prospective controlled clinical study. Surg Endosc. 2004;18(6):919-23.

15. Gurusamy KS, Samraj K, Davidson BR. Low pressure versus standard pressure pneumoperitoneum in laparoscopic cholecystectomy. Cochrane Database Syst Rev. 2009;(2):CD006930.

16. Pang CK, Yap J, chen PP. The effect of an alveolar recruitment strategy on oxygenation during laparosocopic cholecystectomy. Anaesth Intensive Care. 2003;31(2):176-80.

17. Gonzáles Ruiz V, Marenco Correa CA, Chávez Gomes A y col. Colecistectomia laparoscópica: resultados de la experiencia del Hospital General de México a nueve años de implementada. Rev Mex Cir Endosc. 2002;3:71-3.
18. Smith I. Anestesia para laparoscopia con énfasis en el procedimiento en pacientes externos. Clin Anest NA. 2011;1:19-37.

19. Gramatica L, Brasesco OE, Mercado Luna A, Martinessi V, et al. Laparoscopic cholecystectomy performed under regional anaesthesia in patients with chronic obstructive pulmonary disease. Surg Endosc. 2002;16:472-5.

20. Collins LM, Vaghadia H. Regional anaesthesia for laparoscopy. Anesthesiol Clin North America. 2001;19:43-55.

21. López-Herranz GP. Cirugia laparoscópica y anestesia en pacientes de alto riesgo. Rev Med Hosp Gen Mex. 2006;69:164-70.

22. Feig BW, Berger DH, Dougherty TB, Dupvis JF, His B, Hickey RC, et al. Pharmacologic intervention can reestablish baseline hemodynamic parameters during laparoscopy. Surgery. 1994;116:733-41.

23. Bickel A, Eitan A, Melnik D, Weiss A, Gavrieli N, Kniaz D, Intrator $N$. The use of pneumoperitoneum during laparoscopic surgery as a model to study pathophysiologic phenomena: the correlation of cardiac functionality with computerized acoustic indices--preliminary data. J Laparoendosc Adv Surg Tech A. 2012;22(4):349-54.

24. Bickel A, Trossman A, Kukuev I, Eitan A. The effects of high-frequency jet ventilation (HFJV) on pneumoperitoneum-induced cardiovascular changes during laparoscopic surgery. Surg Endosc. 2011;25(11):3518-25.

25. Kim EJ, Yoon H. [The effects of pneumoperitoneum on heart rate, mean arterial blood pressure and cardiac output of hypertensive patients during laparoscopic colectomy]. J Korean Acad Nurs. 2010;40(3):433-41.

26. Cakmakkaya OS, Kaya G, Altintas F, Hayirlioglu M, Ekici B. Restoration of pulmonary compliance after laparoscopic surgery using a simple alveolar recruitment maneuver. J Clin Anesth. 2009;21(6):422-6.

27. Suh MK, Seong KW, Jung SH, Kim SS. The effect of pneumoperitoneum and Trendelenburg position on respiratory mechanics during pelviscopic surgery. Korean J Anesthesiol. 2010;59(5):329-34.

28. Nesek-Adam V, Mrsić V, Smiljanić A, Oberhofer D, Grizelj-Stojcić E. [Pathophysiologic effects of $\mathrm{CO}_{2}-$ pneumoperitoneum in laparoscopic surgery]. Acta Med Croatica. 2007;61(2):165-70.

29. Khoury W, Schreiber L, Szold A, Klausner JM, Wienbroum AA. Renal oxidative stress following $\mathrm{CO} 2$ pneumoperitoneum-like conditions. Surg Endosc. 2009;23(4):776-82.

30. Wiesenthal JD, Fazio LM, Perks AE, Blew BD, Mazer D, Hare G, Honey RJ, Pace KT. Effect of pneumoperitoneum on renal tissue oxygenation and blood flow in a rat model. Urology. 2011;77(6):1508.e9-15.

31. Nathanson LK, Brunott N, Cavallucci D. Adult esophagogastric junction distensibility during general anesthesia assessed with an endoscopic functional luminal imaging probe (EndoFLIP ${ }^{\circledR}$ ). Surg Endosc. 2012;26(4):1051-5.

32. Moncure M, Salem R, Moncure K, Testaiuti M, Marburger R, YeX, Brathwaite C, Ross SE. Central nervous system metabolic and physiologic effects of laparoscopy. Am Surg. 1999;65(2):168-72.

33. Hoekstra LT, Ruys AT, Milstein DM, van Samkar G, van Berge Henegouwen MI, Heger M, Verheij J, van Gulik TM. Effects of prolonged pneumoperitoneum on hepatic perfusion during laparoscopy. Ann Surg. 2012 Jul 20. [Publicación electronic antes de la impresión].
34. Kashefi P, Montazeri K, Honarmand A, Safavi M, Hosseini HM. The analgesic effect of midazolam when added to lidocaine for intravenous regional anaesthesia. J Res Med Sci. 2011;16(9):1139-48.

35. Jabalameli M, Honarmand A, Safavi M, Chitsaz M Treatment of postoperative nausea and vomiting after spinal anesthesia for cesarean delivery: A randomized, double-blinded comparison of midazolam, ondansetron, and a combination. Adv Biomed Res. 2012;1:2.

36. Heidari SM, Talakoub R, Yaraghi Z. Comparing the preventive effect of midazolam and midazolamdexamethasone on postoperative nausea and vomiting in elective middle ear surgery. Adv Biomed Res. 2012;1:9.

37. Choi SH, Kim SH, Lee SJ, Soh SR, Oh YJ. Cerebral oxygenation during laparoscopic surgery: jugular bulb versus regional cerebral oxygen saturation. Yonsei Med J. 2013;54(1):225-30.

38. Dewhirst E, Lancaster C, Tobias JD. Hemodynamic changes following the administration of propofol to facilitate endotracheal intubation during sevoflurane anesthesia. Int J Clin Exp Med. 2013;6(1):26-9

39. Petrenko AB, Yamakura T, Kohno T, Sakimura $\mathrm{K}$, Baba $\mathrm{H}$. Increased brain monoaminergic tone after the NMDA receptor GluN2A subunit gene knockout is responsible for resistance to the hypnotic effect of nitrous oxide. Eur J Pharmacol. 2012 Nov 2. doi:pii: S0014-2999(12)00903-X. 10.1016/j. ejphar.2012.10.034. [Publicación electronic antes de la impresión].

40. Victorzon M, Peromaa-Haavisto P, Tolonen P. Perioperative morbidity, mortality and early outcome of the first 360 gastric bypass operations performed in a district hospital. Scand J Surg. 2012;101(3):184-9.

41. Tsai HW, Chen YJ, Ho CM, Hseu SS, Chao KC, Tsai SK, Wang PH. Maneuvers to decrease laparoscopy-induced shoulder and upper abdominal pain: a randomized controlled study. Arch Surg. 2011;146(12):1360-6.

42. Chatti C, Corsia G, Yates DR, Vaessen C, Bitker MO, Coriat P, Rouprêt M. [Prevention of complications of general anesthesia linked with laparoscopic access and with robot-assisted radical prostatectomy]. Prog Urol. 2011;21(12):829-34.

43. Ulsenheimer K. [Legal liability problems in outpatient operations. View from an anesthesiological perspective]. Anaesthesist. 2012;61(2):156-62.

44. Chatti C, Corsia G, Yates DR, Vaessen C, Bitker MO, Coriat P, Rouprêt M. [Prevention of complications of general anesthesia linked with laparoscopic access and with robot-assisted radical prostatectomy]. Prog Urol. 2011;21(12):829-34.

45. Park EY, Kwon JY, Kim KJ. Carbon dioxide embolism during laparoscopic surgery. Yonsei Med J. 2012;53(3):459-66.

46. Critchley LA, Ho AM. Surgical emphysema as a cause of severe hypercapnia during laparoscopic surgery. Anaesth Intensive Care. 2010;38(6):1094-100.

Artículo recibido el 7 de julio de 2012 y aceptado para publicación el 1 de octubre de 2012.

\section{Correspondencia:}

Dr. Jorge Enciso Nano

Celular: 999305608

Correo electrónico: jorgeencisonano@gmail.com 